\title{
Efficient Learning Management Tool for Web-based Education using an Evolutionary Algorithm
}

\author{
P.Arumugam ${ }^{1}$, Dr.T.Krishnakumar ${ }^{2}$, Dr.M.Sriram ${ }^{3}$ \\ \{ ararun513@gmail.com ${ }^{1}$,drkk@bharathuniv.ac.in ${ }^{2}$, msr1sriram@gmail.com ${ }^{3}$ \} \\ Research Scholar, CSE, Bharath Institute of Higher Education and Research, Chennai, India ${ }^{1}$, \\ Professor, CSE, Bharath Institute of Higher Education and Research, Chennai, India. ${ }^{2}$, Assistant \\ Professor, Department of CSE, Bharath Institute of Higher Education and Research, Chennai ${ }^{3}$
}

\begin{abstract}
The goal of Effective Web based E-Learning is to connection the space among the presentlygeneral technique to Web-based totally education, that is targeted on getting to know control systems (LMS). The influential however underused technologies in smart teaching and adaptive hypermedia. Ontology have end up a key era for permitting semantic-driven resource control. We present a sub ontology-based totally method for resource reuse by way of the use of an Evolutionary set of rules..
\end{abstract}

Keywords: Web based E-Learning, Efficient Learning Management Tool, Evolutionary Algorithm.

\section{Introduction}

A key, general objective for some dedicated instructor is to guarantee that the student has a significant and important cultureinvolvement while accomplishing the ideal culture results. In this tabloid it is contended that to accomplish such an objective, a technique should be set up that is equipped for giving understudies a completely integrated[3], comprehensive learning climate. The thinking, just, is that taking in won't really exude from one explicit basis and when it occurs, it will happen through various methods, for various individuals. One of the extraordinary qualities of the web based knowledge space is that, saddling the force of the different data and correspondence innovations (ICTs), there is more noteworthy extension for cooking for singular adapting needs. In view of this, the paper advances a system that contains various covering 'sub-conditions' which, together, give the platform thought about vital development really comprehensive knowledge climate.

Learning Management System (LMS) - an assortment of e-Learning devices accessible finished common authoritative line. A culture the board framework can be considered as the stage online sequences or sectionsonline sequences gathered and utilized since. 


\section{Semantic Web Views}

The objective semantic web create empowering guidelines and advancements intended to assist machineries with understanding data on the web so they can uphold more extravagant revelation, information joining, route, and mechanization of errands.

With semantic web we not just get more accurate outcomes while looking for Information, yet in addition know when we can incorporate data from various causes, Know what data to think about, and can give a wide range of mechanized administrations in various areas from future home and advanced public library to electriccommercial and Health service [4].

\section{Ontology Views}

Metaphysics arethe record or documentwhich officially characterizes the relation among the footings. The primary use for ontology are to upgrade the precision of web look. The journalists additionally characterize anything a URL and URI are capacities semantic web. URL's is most recognizable sort URI.

Ontologycomprise a fundamental and significant piece Semantic Web. emantic Web prosper, designers should make and coordinate various ontology, from general high level ontology, to area explicit and task-explicit ontology. first inspirations driving philosophy research was the conviction that ontology can assist with reclaim in information portrayal.

\section{Existing System}

Current work for Semantic-Web-or cosmology-based e-learning will in general utilize ontology or semantic model statical to intercede e-learning assets and recover e-learning practices. e-learning framework need to create significant assets composed to accomplish onrequest and community-oriented e-learning Web. In any case, happensvaried portrayal issue to different e-learning assets Web. 


\section{ARCHITECTURE}

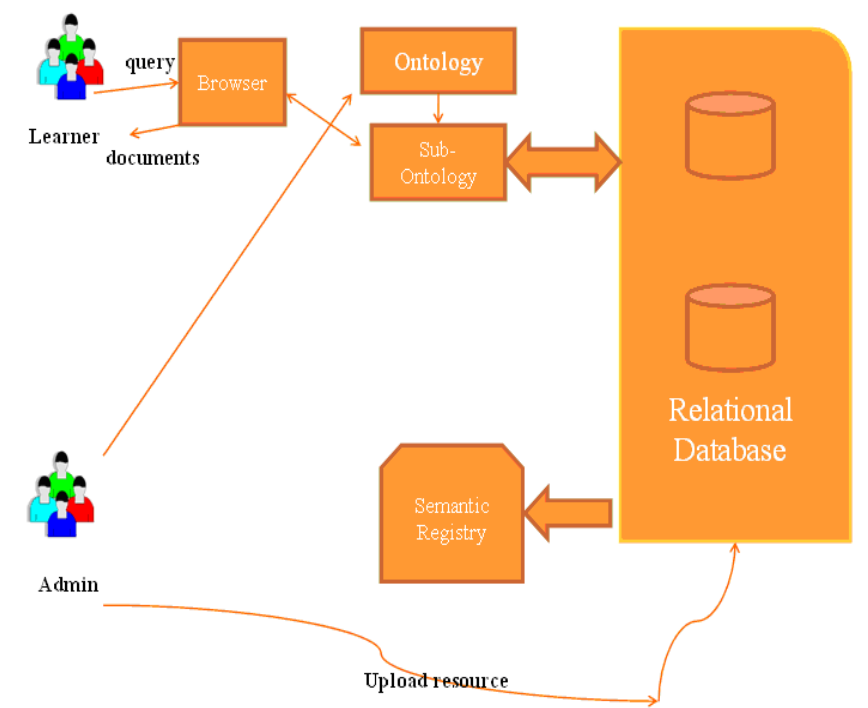

\section{Problem Representation}

As sort of transformative calculation, the hypothetical establishments of GA were initially future by Hollands in the mid 1970 . It smears a portion of the common advancement standards like hybrid, transformation, and natural selection to enhancement and erudition. GA practical numerous issues improvement and characterization. WeadoptGA take care of issue of SubO development to help active asset the executives and recycle. For planning our concern to the GA definition, two stages are should have been achieved, to be specific, issue encoding and deciding the assessment/wellness work dependent on the philosophy semantic.

\section{Evolution Algorithm}

In light of the issue encoding and the wellness work, suggest a calculation for SubO advancement. The accompanying calculation advances a bunch of SubOs dependent on a bunch of asset demands and gets another arrangement of SubOs for asset recycle.

\section{Calculation: Sub Evolve(K, R, O) STEPS}

The advancement calculation is clarified as follow:

1. Recover distinctive SubOs from an archive or concentrate diverse SubOs from the source metaphysics as per distinctive asset demands. 
2. Encode SubOs as a populace of chromosome.

3. Advance the populace dependent on GA.

4. Analyze the chromosome in the populace and union one with high closeness.

5. Assess the chromosome in the populace. End if the general wellness is higher than an edge esteem. Something else, go to stage 3.

6. Interpret the chromosome in the populace to SubOs and reappearance another arrangement of SubOs.

7. Recover e-learning assets identified with the SubOs.

We can recover the asset the board and recycle for e-learning frameworks dependent on the SubO development calculation. The nearby information construction of e-learning frameworks turns out to be more versatile to asset demands through SubO development.

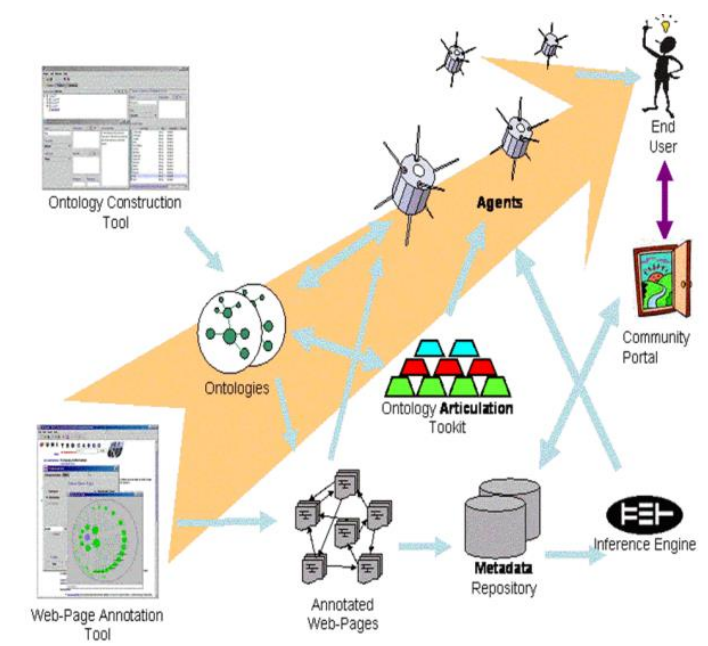

Use of metadata on the Semantic Web

\section{Conclusion}

We suggest a powerful SubO component versatile administration and recycle of elearning assets disseminated climate the Web. That contend that to accomplish -request semantics-based asset the board for Web-based e-learning, one ought to go past utilizing area ontology stoical. We suggest a semantics planning system to incorporate e-learning data sets by utilizing philosophy semantic. 
Characterize setting explicit parts from the entire philosophy SubOs and suggest a SubObased asset reclaim method by utilizing an advancement calculation. Clarify the GA-based development calculation active e-learning asset reclaim element. Additionally lead a reproduction try and assess the suggested method TCM e-learning situation. The suggested SubO-based methodology for e-learning asset the executives and reclaim is a long way from existence adult.

\section{References}

[1] M. Nichols and D. Schedule, "A Theory for eLearning," Educational Technology and Soc., vol. 6, no. 2, pp. 1-10, 2003.

[2] T. Berners-Lee, J. Hendler, and O. Lassila, "The Semantic Web," Scientific Am., vol. 284, no. 5, pp. 34-43, 2001.

[3] Dr.Kaliyamurthie K.P “An Application Of Non-Uniform Cellular Automata For Efficient Cryptography”, Indian Journal of Science and Technology, Vol 6, Issue 5S, page 4648-4652 May 2013.

[4] Dr. Kaliyamurthie K.P "K-Anonymity Based Privacy Preserving For Data Collection In Wireless Sensor Networks", Indian Journal of Science and Technology, Vol 6, Issue 5S, page 4604-4614 May 2013.

[5] Dr. Kaliyamurthie K.P "Highly Secured Online Voting System Over Network", Indian Journal of Science and Technology, Vol 6, Issue 6S page 4831-4836 May 2013.

[6] Dr. Kumaravel. A "Vehnode: Wireless Sensor Network Platform For Automobile Pollution Control” IEEE explore, Vol Page(s): 963 - 966, 2013.

[7] Dr. Kumaravel. A "Multi- Classification Approach For Detecting Network" IEEE explore, Page(s): 1114 -1117,April 2013.

[8] T. Vijayan , M. Sangeetha , A. Kumaravel \& B. Karthik (2020): FeatureSelection for Simple Color Histogram Filter based on Retinal Fundus Images for DiabeticRetinopathy Recognition, IETE Journal of Research, DOI: 10.1080/03772063.2020.1844082.

[9] D. S. Vijayan, A. Leema Rose, S. Arvindan, J. Revathy, C. Amuthadevi, “Automation systems in smart buildings: a review", Journal of Ambient Intelligence and Humanized Computing https://doi.org/10.1007/s12652-020-02666-9

[10] Vijayan T, Sangeetha M, A. Kumaravel, Karthik B, "Gabor filter and machine learning based diabetic retinopathy analysis and detection", Microprocessors and Microsystems,2020. https://doi.org/10.1016/j.micpro.2020.103353.

[11] Vijayan T, SangeethaM, Karthik B, "Trainable WEKA Segmentation of Retinal Fundus Images for Global Eye Disease Diagnosis Application,” International Journal of Emerging Trends in Engineering Research,Vol 8, No.9, pp. 5750-5754, Sep 2020. https://doi.org/10.30534/ijeter/2020/136892020

[12] C. Amuthadevi, D. S. Vijayan, Varatharajan Ramachandran, "Development of air quality monitoring (AQM) models using different machine learning approaches", Journal of Ambient Intelligence and Humanized Computing, https://doi.org/10.1007/s12652-020-02724-2

[13] Vijayan T, Sangeetha M, A. Kumaravel, Karthik B, "Fine Tuned VGG19 Convolutional Neural Network Architecture for Diabetic Retinopathy Diagnosis," Indian Journal of Computer Science and Engineering (IJCSE), Vol. 11, No. 5, pp. 615-622 Sep-Oct 2020. DOI: 10.21817/indjcse/2020/v11i5/201105266.

[14] Vijayan T, Sangeetha M, Karthik B, "Efficient Analysis of Diabetic Retinopathy on Retinal Fundus Images using Deep Learning Techniques with Inception V3 Architecture," Journal of Green Engineering, Vol 10, Issue 10, pp. 9615-9625. Oct 2020 